\title{
Malestares y enfermedades entre líneas: hombres que narraron sus experiencias en la paternidad
}

\section{Ailments and illnesses between the lines: men recounting their experiences in fatherhood}

Yeimi Alejandra Colín Paz ${ }^{a}$

\begin{abstract}
:
This article recovers the experiences of some men, in relation to their being a man and fatherhood, analyzing the possible relationship between these elements and health and disease. The purpose is to include men's health on the public policy agenda and, among them, those who are fathers. Not only because in Mexico there is a higher incidence of deaths of men than of women at productive and reproductive ages, but because by making them visible, behaviors and attitudes that affect people close to men could be rethought.
\end{abstract}

Keywords:

fatherhook, helth, disease, public politics

\section{Resumen:}

En este artículo se recuperan las experiencias de algunos varones, en relación a su ser hombre y la paternidad, analizando la posible relación entre estos elementos y la salud y enfermedad. El propósito es incluir en la agenda de las políticas públicas la salud de los varones y entre ellos, los que son padres. No solo porque en México hay una mayor incidencia de muertes de hombres que de mujeres en edades productivas y reproductivas, sino porque al visibilizarlos se podrían replantear comportamientos y actitudes que afectan a las personas cercanas a los varones.

\section{Palabras Clave:}

Paternidad, salud, enfermedad, políticas públicas

\section{Introducción}

La forma en que se vivencian las masculinidades afecta las condiciones de salud de los hombres. Algunos autores sustentarían esta aseveración, como De Keijzer (1997), quien sugiere que la masculinidad es un factor de riesgo para los hombres y para otras personas", en virtud de las conductas o masculinidades enajenadas que muchos adoptan y reproducen dados los aprendizajes de género. Es decir, debido a la falta de elementos para expresar sus emociones o derivado de su obstinación (voluntaria o impuesta), por la temeridad a fin de probar valentía, fortaleza, poderío y virilidad. Tena (2007) ha señalado, que el "deber ser proveedor y jefe económico del hogar", con el propósito de probar el éxito masculino o consumar el dominio de los hombres sobre otros, ha nutrido la idea generalizada del trabajo como una actividad de competencia masculina, que en ocasiones se realiza en condiciones adversas y factibles al deterioro de la salud, promoviendo, por ejemplo, la ocurrencia del burnout (Tena, 2014). En tanto que Bolaños (2014) documenta al desempleo, como nocivo para los varones, al sentir cuartada la posibilidad de cumplir con los preceptos de la masculinidad socialmente esperada.

Figueroa (2015) ha dedicado parte de sus reflexiones en este mismo sentido, mostrando la existencia de una mayor incidencia de enfermedades y muertes en México de hombres que de mujeres, pero además invita a los investigadores de diversas disciplinas al diálogo para tratar la enfermedad y muerte paterna, por

\footnotetext{
* Mujeres, niñas, niños y otros hombres con menos recursos de poder.
}

a Consultora Independiente, https://orcid.org/0000-0002-7745-9552, Email: yeimi.colin.paz@colmex.mx 
analogía al riesgo de salud y mortalidad materna. De tal forma que, si las mujeres-madres pueden cursar por problemas de salud al grado de verla disminuida durante el embarazo, parto y puerperio y/o perder la vida en el proceso; los varones progenitores pudieran deteriorar la salud y la vida debido a situaciones asociadas con la paternidad (Figueroa, 2005, 2011, 2014 y 2014a). Por eso incluso, ha propuesto las autopsias verbales para analizar casos por suicidio en varones progenitores (Nájera y Figueroa, 2015).

Los estudios que vincula la paternidad con la salud, enfermedad y muerte de los varones son poco convencionales en México e incluso controvertidos, dado el tiempo que le ha llevado a las activistas y feministas, documentar y hacer reconocer el derecho de las mujeres a la salud sexual y reproductiva, si bien es sabido, por la Legislación Nacional y particularmente por el Art. 4to. de la Constitución Política Mexicana que, mujeres y hombres tienen derecho a decidir de manera libre, responsable e informada, el número y espaciamiento de sus hijos (SS, 2020).

El presente artículo, tiene como propósito mostrar algunas experiencias de varones en el espacio de la paternidad $^{*}$ y su posible relación con la salud, en virtud de contribuir al conocimiento del tema analizando y dialogando sobre posibles áreas de atención para la generación de política pública en favor de la salud masculina y paterna, las que actualmente están ausentes en la agenda pública y social. Si bien es fundamental, no únicamente porel derecho de todos a recibir servicios que nos permitan mantenernos saludables, sino porque al invisibilizar a los varones y entre ellos a los papás, se afecta también a los miembros de la familia y a otras personas cercanas a su entorno, como resultado de la reproducción de comportamientos, prácticas y roles asociados a los aprendizajes de género masculinos y de la paternidad, construidos socialmente e incorporados durante el desarrollo cotidiano de los hombres.

\section{Método}

Se realizaron ocho entrevistas a profundidad con varones residentes en la Ciudad de México, que tenían en común haber tenido hijos y que tuvieran entre 20 y 50 años. El proyecto forma parte de otro presentado para obtener el grado doctoral en 2018, y se construyó a partir de un paradigma cualitativo. Es decir, no se priorizó buscar características particulares de los progenitores o sus descendientes para encontrar patrones de

\footnotetext{
* Los casos que se muestran en este artículo formaron parte del proyecto doctoral "Salud, enfermedad y muerte de algunos varones que viven o vivieron la experiencia de la paternidad en la Ciudad de México", concluida y presentada en el año 2018.

$\dagger$ En septiembre de 2017 ocurrieron tres catástrofes naturales en la Ciudad de México que impidieron a uno de los informantes
}

comportamiento inherentes, sino que se intentó indagar y dialogar un tema no explorado y que sugiere una representatividad por transferibilidad. Esto es, mediante un análisis detallado del contexto y características de cada sujeto de estudio, de tal forma, que se pueda inferir que otros individuos en condiciones y situaciones similares, tendrían mayor posibilidad de incursionar porel fenómeno en estudio (Martínez, 2012), siendo el caso aquí, de la salud y/o la enfermedad asociada a la paternidad.

Existe un antecedente teórico que suscribe, que los hombres tienen dificultades para expresar sus emociones y padecimientos, además de encontrarse reacios a solicitar apoyo de familiares, amigos y personal de salud cuando sienten disminuida su salud (Colín, 2018; Figueroa, 2014b; Evans, et. al., 2011; Núñez, 2004; Torres, 2002; Kaufman, 1997). Por tal razón, en el acercamiento con los progenitores sólo se les comentó que se intentaba indagar sobre las experiencias alrededor del ser hombre y la paternidad, reservándome el objetivo de búsqueda de elementos asociados con la salud y enfermedad. Esto tuvo propósito, lograr que los participantes se sintieran libres y en confianza de conversar sobre cualquier tema relacionado a su paternidad, sin que sintieran cuestionada su virilidad o masculinidad.

Inicialmente, se les pidió a los varones participantes autorización para ser entrevistados comentándoles los objetivos generales del estudio; se hizo el compromiso de protección de identidad; además que si alguna pregunta resultaba incómoda tenían la libertad de reservarse la respuesta o renunciar definitivamente a continuar participando. Asimismo, se ofreció conversar con un profesional de la salud emocional, si durante la entrevista se propiciaban recuerdos dolorosos o difíciles de elaborar; esto con el propósito de que se sintieran acompañados y también para evitar un daño emocional inherente a la entrevista.

Para obtener la muestra, se lanzó una convocatoria por redes sociales (Facebook y Twiter), para encontrar varones que cumplieran con las tres condiciones. A ella, respondieron las personas cuyas características se detallan en el cuadro 1.

Las entrevistas comenzaron a realizar desde diciembre de 2016 y durante 2017. Inicialmente se tenían cinco entrevistas, pero la de Leonardo no pudo ser concluida $^{\dagger}$ (aunque fue incluida en esta investigación dada la riqueza de información), así que se decidió ampliar la muestra, con hombres que tuvieran características que

completar la entrevista. Una inundación y un terremoto solo retrasaron la entrevista, pero el último terremoto destruyó un edificio contiguo a la residencia del informante y ya no pudo participar. 
resultaron interesantes en Leonardo y se integraron entonces Horacio, que cumplía con las condiciones básicas y por su deseo de participar; Diego, porque contaba con hijos adolescentes y Julio, que además de tener una hija de mayor edad, también se encontraba en el límite máximo de la edad (50 años).

Cuadro 1.

Características de los Sujetos de Estudio

\begin{tabular}{|c|c|}
\hline Nombre & Características \\
\hline \multirow{10}{*}{ Bruno } & - 29 años \\
\hline & - Pasante de ingeniería \\
\hline & - Imparte clases particulares y servicios \\
\hline & didácticos en escuelas particulares \\
\hline & - Vive en unión libre desde hace un año y \\
\hline & medio \\
\hline & - Tiene una hija de un año \\
\hline & - Ingreso mensual de 5 mil pesos \\
\hline & - Pareja tiene 28 años, licenciatura y percibe \\
\hline & 14 mil pesos mensuales \\
\hline \multirow{7}{*}{ Claudio } & - 38 años \\
\hline & - Arquitecto y estudia un posgrado \\
\hline & - Es docente de universidad, asesor \\
\hline & inmobiliario y baterista \\
\hline & - Casado pero separado \\
\hline & - Tiene 2 hijos varones de 8 y 11 años \\
\hline & - Su exesposa tiene una licenciatura y trabaja \\
\hline \multirow{14}{*}{ Diego } & - 43 años \\
\hline & - Sociólogo \\
\hline & - Empleado de Gobierno \\
\hline & - Soltero (divorciado) y vive con una pareja \\
\hline & - Tiene 3 hijos del primer matrimonio (17 el \\
\hline & varón y 2 chicas de 16 y 13 años) y dependen \\
\hline & económicamente de él. \\
\hline & - Percibe 40 mil pesos mensuales \\
\hline & - Exesposa: tiene 41 años. Ama de casa, \\
\hline & $\begin{array}{l}\text { parece que eventualmente tiene trabajos no } \\
\text { fijos y tiene licenciatura. }\end{array}$ \\
\hline & - Pareja actual: tiene 45 años, estudió una \\
\hline & maestría y trabaja. Ingreso mensual de 25 a \\
\hline & 30 mil pesos. Tiene una hija de 17 años que \\
\hline & $\begin{array}{l}\text { vive con ellos (no es dependiente económica } \\
\text { de Diego). }\end{array}$ \\
\hline \multirow{7}{*}{ Fernando } & - 44 años \\
\hline & - Politólogo \\
\hline & - Es profesor y subdirector en una secundaria \\
\hline & - Casado \\
\hline & - 1 hijo de 12 años \\
\hline & - Ingreso mensual de 16 mil pesos \\
\hline & $\begin{array}{l}\text { - Esposa: } 44 \text { años, licenciada y trabaja, } \\
\text { sueldo: } 18 \text { mil pesos mensuales }\end{array}$ \\
\hline \multirow{6}{*}{ Gerardo } & - 44 años \\
\hline & - Sociólogo \\
\hline & - Consultor \\
\hline & - Casado \\
\hline & - Tiene 1 hijo de 6 años \\
\hline & - Ingreso mensual entre 20 y 30 mil pesos \\
\hline
\end{tabular}

\begin{tabular}{|c|c|}
\hline & $\begin{array}{l}\text { - Esposa: } 44 \text { años, con licenciatura. Ingreso } \\
\text { mensual de } 20 \text { a } 25 \text { mil pesos }\end{array}$ \\
\hline Horacio & $\begin{array}{l}\text { - } 45 \text { años } \\
\text { - Ingeniero } \\
\text { - Se dedica a la tecnología de la información } \\
\text { y diseño de servicios } \\
\text { - Unión libre desde hace } 14 \text { años } \\
\text { - Tiene } 2 \text { hijos varones de } 7 \text { y } 2 \text { años } \\
\text { - Ingresos variables (en promedio } 50 \text { a } 60 \text { mil } \\
\text { pesos mensuales) } \\
\text { - Pareja: } 41 \text { años, licenciada y trabaja por } \\
\text { entre } 22 \text { y } 25 \text { mil pesos mensuales. }\end{array}$ \\
\hline Julio & $\begin{array}{l}\text { - } 50 \text { años } \\
\text { - Pedagogo } \\
\text { - Es andragogo (pedagogo que trabaja con } \\
\text { adultos) y docente } \\
\text { - Divorciado dos veces } \\
\text { - Tiene una hija de } 20 \text { años del primer } \\
\text { matrimonio } \\
\text { - Primera esposa: } 49 \text { años y escolaridad de } \\
\text { secundaria } \\
\text { - Segunda esposa: } 46 \text { años y un doctorado, } \\
\text { trabaja en la academia. } \\
\text { - Ingreso mensual de } 11,500 \text { pesos } \\
\text { - Residencia: Guanajuato }\end{array}$ \\
\hline Leonardo & $\begin{array}{l}\text { - } 50 \text { años } \\
\text { - Filósofo y Abogado, aunque de la primera } \\
\text { no tiene título } \\
\text { - Hace servicios notariales } \\
\text { - Divorciado y casado actualmente } \\
\text { - Tiene } 3 \text { hijos, un varón de } 19 \text { años del primer } \\
\text { matrimonio y } 2 \text { hijos (hombre y mujer de } 12 \text { y } \\
11 \text { años respectivamente) del segundo } \\
\text { matrimonio. } \\
\text { - Exesposa: } 48 \text { años, trabaja y tiene } \\
\text { licenciatura. } \\
\text { - Esposa actual: } 40 \text { años, hizo una carrera } \\
\text { técnica, no ejerce porque se dedica al hogar. } \\
\text { Ayuda a Leonardo a hacer trámites de su } \\
\text { trabajo y estudia una licenciatura en línea. } \\
\text { - No especifica su ingreso mensual porque es } \\
\text { variable, pero necesita al menos } 40 \text { mil } \\
\text { pesos. }\end{array}$ \\
\hline
\end{tabular}

Fuente: Colín (2018)

\section{Resultados}

Los hombres narraron algunas experiencias paternas, quizá sin reparar en que estaban nombrando malestares y/o padecimientos sentidos y vividos en el entorno de la paternidad, ya que a pesar de percibirlos a través de su cuerpo o en relación con él, no han aprendido a identificarlos, nombrarlos, tratarlos y menos aún a evitarlos, a propósito de que estos detonen enfermedades, accidentes o agravamiento de padecimientos previos e incluso que fomenten la posibilidad de morir. Además, los comportamientos y 
actitudes empleados para evadir los malestares o cuidar de su salud lejos de ayudar, pudieran acentúan o promover prácticas poco saludables y más dañinas.

Inicialmente, recupero lo que dijeron los progenitores respecto a su sentimiento de salud al momento de la entrevista. Después describiré lo que desconocen que forma parte de ella, que también verbalizaron y que parece simbolizar padecimientos, malestares y tensiones físicas, emocionales o quizá hasta enfermedades aun no clasificadas oficialmente como tal. Posteriormente, se abordan los malestares que pudieran asociarse a la construcción del género masculino y a la paternidad, en virtud de dialogar y valorar su importancia en la generación futura de políticas públicas en salud para los varones y padres.

\section{Salud percibida}

Antes de dar a conocer las respuestas de los participantes, es necesario señalar, que el significado de salud pudo ser entendido de diversas formas, pues el concepto de la OMS que es el más vigente y reconocido internacionalmente, es muy general y la define como "el completo estado de bienestar físico, mental y social y no solo como la ausencia de afecciones o enfermedades" (OMS, 2021), lo que supone poder definir que es bienestar y si aplica para toda la población en diferentes espacios y tiempos o si es un estado de condiciones positivas $u$ óptimas, sin advertir lo intrínseco de la enfermedad o de los contextos adversos. En este sentido, habría que saber que los participantes pudieron entenderla, como la ausencia de enfermedad, la sensación física del malestar o bienestar, la inclusión de la emocionalidad, el acceso a los servicios clínicos por prevención y atención o algo que incluye todas las opciones.

Dicho lo anterior, cuatro varones dijeron encontrarse bien de salud. Aunque enunciaron a la par, malestares o enfermedades que padecían desde tiempo atrás y que sobrellevaban de la mejor manera o con dificultad, pero con resiliencia. Lo que se parece más a la definición de salud de Canguilhem, quien la suscribe como la capacidad humana de no estar enfermo, recuperarse de distintas enfermedades $y / 0$ adaptarse a vivir con una o varias de ellas, procurando realizar sus actividades cotidianas y evitando deteriorar más el cuerpo biopsicosocial (Le Blanc, 2004; Caponi, 1997; Martínez, 2008).

Pero la respuesta de estos cuatro hombres también pudiera simbolizar, la réplica de los aprendizajes del género masculino que les conmina a no evidenciar sus vulnerabilidades y sus sentimientos o a mostrar control de contexto a pesar de las adversidades (Colín, 2018; Evans, et. al., 2011).
Así pues, Bruno, Fernando, Gerardo y Julio dijeron sentirse bien de salud, pero mostraron inconformidad con algún aspecto de su vida.

Bruno, no se sentía satisfactorio con su cueno (tenía sobrepeso), con su vida (creía haber desperdiciado oportunidades importantes como concluir la universidad) y tampoco estaba a gusto con su vida sexual (dado que su esposa viaja constantemente por razones de trabajo). Aunque, por otro lado, había condiciones que lo hacían sentirse sumamente orgulloso, como ser papá, ejercer y aprender de su paternidad, apoyar a su pareja, familia y a otras personas cercanas. Adicionalmente, tenía elevado el ácido úrico, lo que no se denomina como enfermedad, pero que produce malestares y vulnera al cuerpo, al grado de ponerlo en peligro de muerte.

Fernando por su parte, se sentía bien con su cuerpo, con su vida, estaba orgulloso de él y se consideró muy útil para sí, su familia y las personas que le rodeaban. No obstante, vivía permanentemente con colitis y gastritis, además de tener síndrome de colon irritable y rinitis alérgica, padecimientos que en muchas ocasiones le complican la posibilidad de cumplir con sus actividades cotidianas.

Gerardo igualmente señaló estar sano, a pesar de tener permanente colitis, gastritis y un pie que se le iba enchuecando con el paso de los años. Se sabía en sobrepeso, consideraba tener una actividad laboral ociosa y una vida sexual "costumbrista" (sic) que le gustaría diversificar, pero no lo hace por evitar conversarlo con su pareja, pues dice, no es un punto prioritario de su relación. Julio, parecía haberse valorado como saludable, al comparar su sentir con la cantidad de enfermedades y padecimientos que experimentaba, pues cursaba por las secuelas de un Evento Vascular Cerebral (EVC), además de tener síndrome metabólico, hipertensión, colitis, gastritis, depresión mayory otros padecimientos de menor envergadura como: astigmatismo y no sentirse muy satisfecho con su cuerpo (por obesidad) o con su salud sexual, pues no tenía pareja y la única actividad aunque satisfactoria, era la masturbación.

Claudio, Horacio y Leonardo, en contraste, matizaron su condición de salud y la evaluaron como regular. Sin embargo, experimentaron malestares muy similares a los participantes anteriores.

Claudio se consideró con sobrepeso; se sabía con presión arterial alta (con propensión a la hipertensión); problemas de hígado, pues no procesaba adecuadamente las grasas y le salieron bolitas en los brazos y las piernas; también le brotaron manchas blancas en la piel, producto de tensiones o el manejo inadecuado del estrés. En tanto que emocionalmente, tenía sentimientos de culpa y derrota por no haberse cumplido con las expectativas de sus hijos de mantener la solidez de una familia, si bien pareciera que no cu mplió con su propio proyecto de vida. 
Horacio, recién salía de una crisis por síndrome de colon irritable, lo que lo hacía entran en conflicto con su cuerpo, pues le quitaba energía para realizar otras actividades prioritarias para él. Además, se sabía con sobrepeso, propensión a la hipertensión, problemas permanentes de estrés y un reciente dolor de pierna cuyo origen desconocía.

En tanto que Leonardo, recientemente había consultado al médico, quién le dijo que tenía la presión y el colesterol elevado y diez kilos de sobrepeso. Ese diagnóstico lo conoció, debido a que su esposa lo convención de visitar al médico por una gripe y tos que le duró semanas y por un dolor de cabeza que, según Leonardo se debía a la mala graduación de unos lentes nuevos.

Diego fue el único de los progenitores que abiertamente dijo sentirse muy mal de salud. Si bien sus malestares eran muy parecidos al de los participantes previos (sobrepeso, cansancio, inflamación del estómago, un poco de dolor de cabeza, estrés). No obstante, un dato particular fue la enunciación de inestabilidad con la vida y consigo mismo, debido a una relación poco virtuosa con sus hijos, que le estaba llevando a tomar decisiones equivocadas, como alimentarse inadecuadamente, fumar de más, tomar mucho refresco, sentirse cansado, deprimido, desidioso, pesimista y por eso no realizar actividad física, ni aprovechar las oportunidades que le llegaban.

\section{Salud y paternidad}

Una segunda tarea prioritaria fue encontrar alguna relación entre la salud de los varones con sus vivencias paternas. Dada la escasez de información y/o bibliografía relativa al tema, teoricé con base en los temas más cercanos (salud, enfermedad, estudios de género, masculinidades y la paternidad), a la par de analizar una serie de casos paradigmáticos, que localicé en fuentes de información poco convencionales o menos utilizadas que las fuentes de información formal ${ }^{*}$ y que en términos generales, refieren fragmentos de vida de los varones que pudieron haber enfermado o muerto por alguna condición, situación o práctica asociada a su paternidad.

Como resultado de aquel análisis, categoricé tres tipos de mediaciones intervinientes en la enfermedad o muerte de los varones. La primera y de mayor influencia

\footnotetext{
* Notas periodísticas, historias que fueron contadas de persona a persona, casos que fueron expuestos en libros académicos y personajes literarios, de canciones o de películas.

$\uparrow$ Para mayor detalle sobre el análisis de los casos paradigmáticos ver Colín (2018), Capítulo I. Perspectivas de la cotidianidad de los varones progenitores: casos paradigmáticos.

$\$$ Acto dañino contra uno mismo que aparenta un suicidio, pero sin la determinación de morir, aunque puede terminar con la vida por accidente o descuido. Es una llamada de atención muy seria
}

fue la función como proveedor económico, que al ser la más recurrente y nutrida, tuvo que dividirse en cinco subcategorías: 1) dificultades económicas, 2) cumplimiento del rol como proveedor único o mayoritario, 3) cometer actos ilícitos con el propósito de proveer económicamente a la familia, 4) paternidad ausente por migración e 5) incumplimiento del rol como proveedor. La segunda categoría, se refiere al rol del padre como protector de los descendientes y la tercera categoría, guarda relación con la paternidad interrumpida, ya sea por la separación con los hijos(as), derivado de un divorcio o ante la pérdida (desaparición o muerte) del descendiente ${ }^{\dagger}$.

Los malestares procedentes de estas condiciones no han sido visibilizados desde la disciplina médica, quizá porque como diría Burín (1995, citada por Cantoral, 2014), los malestares son sensaciones subjetivas de padecimientos psíquicos que no se encuentran dentro de los criterios clásicos de la salud o enfermedad, sino que están asociados a nociones como el conflicto o crisis. No obstante, cuando el cuerpo es sometido a sufrimiento o agobio constante y por un periodo de tiempo prolongado, pudiera detonar enfermedades físicas y mentales o la muerte misma, debido a la infiltración de enfermedades diversas ante un cuerpo vulnerado o por actitudes y comportamientos que promueven la ocurrencia de accidentes, suicidios y conductas parasuicidas ${ }^{\ddagger}$ disfrazadas de otras causas violentas de muerte como, por ejemplo, el homicidio.

Es importante mencionar en este punto, que el análisis que se hizo alrededor de las vivencias de los varones en la paternidad no tiene un sustento médico ni clínico, sino que se observó con un lente crítico desde las ciencias sociales y los estudios de población, con el propósito de contribuir al entendimiento y conocimiento del tema, de una manera propositiva, reflexiva y dialógica.

Teniendo esto en cuenta, se en contró que Bruno $\$$ sufrió una parálisis facial, debido quizá a las complicaciones que presentó su hija desde el nacimiento prematuro y hasta los dos años, aunado a la necesidad inherente de continuar generando ingresos para el hogar, a pesar de no ser el proveedor principal, pues él era quien cuidaba a su hija y se encargaba del hogar desde el inicio de la relación conyugal, mientras su esposa trabajaba.

Bruno comentó, que no se sentía del todo útil porque está aprendiendo a ser papa y vivir en pareja, pero no a buscar trabajo o terminar la escuela y eso le causaba

realizada mediante una conducta impulsiva o autodestructiva. Ver: Diccionario de la Real Academia Nacional de Medicina de España http://dtme.ranm.es/terminos/parasuicidio.html?id=160

$\S$ La narrativa completa de la historia de Bruno o de los otros siete participantes de esta investigación, pueden ser consultadas en: Colín, Y. (2018), Salud, enfermedad y muerte de algunos varones que viven o vivieron la experiencia de la paternidad en la Ciudad de México, El Colegio de México. 
tristeza. Igualmente, durante el embarazo, parto y los meses posteriores al nacimiento de su hija se encontró en permanentemente preocupación por la vida de ella, pues los médicos no daban esperanzas. Una vez que su hija superó el peligro de muerte, su atención se centró en la rehabilitación. Ella necesitaba mucha atención y él, a pesar de ser muy paciente, se sentía agotado y agobiado, no tenía tiempo para él mismo, en ocasiones soportaba condiciones de trabajo complicadas con tal de no perderlo; fue asaltado varias veces en el transporte público, incluso con su hija en brazos; hacía ayunos prolongados y no dormía suficiente. En una ocasión, pidió un préstamo al banco para comprar un instrumento que le serviría para su trabajo, asumiendo que el artefacto llegaría a tiempo, adquirió compromisos con clientes, se acercaba el tiempo para cumplir y no llegaba; a la par, llevó a su hija a su consulta cotidiana y el médico le informó que era probable que tuvieran que intervenirla quirúrgicamente una vez más a sus dos años y en ese contexto ocu rrió la parálisis facial.

Claudio, pudo estar padeciendo complicaciones de presión, problemas de hígado y manchas en la piel por estrés como asumía, pero el origen tal vez fue la vulnerabilidad emocional por no poder vivir con sus hijos (más allá de solo convivir). Además, los problemas relacionados con la paternidad pudieron ser detonantes de comportamientos violentos, a pesar de que los consideraba inaceptables y dañinos.

El contexto se presentó cuando ni él ni su novia planearon el embarazo de su primer hijo, no obstante, tuvieron que resolver sus vidas cuando se presentó el caso. No coincidieron en la forma en que convivirían, ella se quería casar y él vivir en unión libre; Claudio optaba por trabajar por cuenta propia y ella porque se empleara en una institución para tener ingresos fijos. Ninguno cedía en la aportación económica, ella pensaba que el hombre debía proveer a pesar de que ambos trabajaban; diferían en el método anticonceptivo a utilizar, el número de hijos a tener y si los hijos asistirían a escuelas públicas o privadas (porque al final tu vieron dos, el segundo tampoco planeado). No congeniaron en el lugar que atenderían su salud (clínicas públicas o privadas), ni el tipo de auto, la ropa que usarían, etc. Con el tiempo, la relación se disolvía y crecían las señales de violencia verbal por ambas partes, no obstante, fue él quien, en un arranque de enojo, ejerció violencia física y entonces se separaron.

Claudio continuaba con el compromiso de pagar las deudas adqu irió el matrimonio, además de cumplircon la manutención de los hijos, que en principio no veía, pues ellos no lo deseaban. Para Claudio, el asunto económico no era un problema, pues siempre tuvo trabajo, el apoyo de sus padres y de la mamá de sus hijos quien trabajaba y podía encargarse de ellos si él no podía aportarles. Su problema era la frustración de no cumplir con sus expectativas de tener una familia; el dolor de no vivir con sus hijos de tiempo completo o la culpa de haber provocado, junto con su expareja, que ellos vivenciaran episodios de violencia que les produjeron miedo.

Otro caso de paternidad con culpa, lo experimentó Leonardo con su primer hijo, a quien asume abandonó pues no soportaba la presencia de la madre. Desde la perspectiva de Leonardo, su exesposa lo manipulaba, era proveedora económica mayoritaria y se apropió de su libertad. Él aportaba todo su sueldo y ella administraba, le decía qué coche usar, qué ropa vestir, a dónde ir de vacaciones e incluso decidió el momento en que procrearían, pues aparentemente había un acuerdo de prevención anticonceptiva para posponer su reproducción. Al experimentar una paternidad impuesta, manipulado y hasta incitado a la violencia doméstica, Leonardo adoptó conductas que pudieran haber puesto en peligro su salud y vida, tuvo relaciones extramaritales y tomó alcohol excesivamente, pues esperaba recuperar la libertad que no había tenido, primero por vivir en un espacio religioso ${ }^{*}$ y luego por involucrarse en el primer matrimonio.

Posteriormente, Leonardo conformó una nueva familia, pero el primogénito segu ía sien do "un aguijón de culpa que lo fastidia”, al no vivir con él, recibió solo la educación de la madre y como se asemeja a ella en actitudes, Leonardo no soporta esa condición, au nque lo ama y sufre por no "rescatarlo", "por cobarde", "por cómodo"y "por querer serfeliz". Su hijo mayor es también, dice, la causa de la mayoría de los conflictos con su esposa, pues aparentemente entorpecía la armonía familiar, al afectar emocionalmente a sus hermanos menores o medios hermanos, pues el primogénito no los reconocía como hermanos. De tal forma que, cuando el hijo mayor tenía problemas con Leonardo, su esposa le exigía que lo alejara de sus hermanos para no afectarlos y esa situación era más dolorosa para Leonardo. La figura de Leonardo en el entorno familiar es muy recia y autoritaria, de tal forma que, ante los problemas, no reaccionaba apaciblemente sino agresivo, impartiendo justicia, aunque a veces cometía injusticias, si bien después reconocía el error y se disculpaba u ofrecía recompensas.

Recordemos que Diego fue el único de los progenitores entrevistados que dijo sentirse muy mal de salud, debido a la relación inestable con sus dos hijas. Lo que no solo se reflejaba en su salud física sino también en el estado emocional, pues se sentía en crisis como papá, después de separarse de ellos, por el divorcio que devino

\footnotetext{
* Leonardo estudió para ser sacerdote, no obstante, conoció a la que fue su primera esposa y pidió una dispensa a la iglesia para no faltarle al respeto a una institución a la que el respetaba.
} 
de una infidelidad de la esposa. Su sensación entonces era de tristeza, preocupación, frustración y depresión, sabiéndose negativo, presionado, pesimista, desidioso, dudoso, retraído, con malos hábitos alimenticios, conduciendo distraído, fumando demasiado, tomando mucho refresco y con pocos ánimos para hacer ejercicio.

Además, no hay que dejar de anotar, que con el ingreso que percibía sostenía a sus tres hijos, a la exesposa y a él, pero también estaba conformando una nueva familia y su propósito era contribuir con los gastos, condición que no había logrado hacer. Esto convertía el problema en un asunto de funciones paternas por proveeduría económica y por paternidad interrumpida por divorcio. Diego también se encontraba molesto por la actitud de sus hijas, quienes se negaban a convivir con él cuando se encontraba con su pareja y por otro lado, no dejaban de exigir o mostrarse groseras si Diego les pedía moderación en sus gastos, pues no tenía forma de generar más recursos.

Los malestares asociados a la proveeduría económica y la paternidad, ya había hecho mella antes del divorcio. Diego compartió que en las fiestas familiares solía beber en exceso, no dormir du rante el fin de semana y después sentirse muy mal. En alguna ocasión, fue a hospitalizarse porque sentía que se le salían las vísceras, le sangraba el recto y pensaba que moriría, pero los médicos le explicaron que era ansiedad y estrés, que Diego asociaba a la falta de recursos para cubrir los gastos de su familia. Ese fue el episodio más importante, pero reconoció que habían ocurrido al menos dos más, que lo espantaron y por ende, lo obligaron a pedir ayuda médica. Si bien, no es un tema que pudiera externar con familiares y amigos, pues no quería preocu parlos, también puede deberse a la creencia de que los hombres no deben exponer sus vulnerabilidades y por el contrario mostrarse fuertes y controlados (Graña, 2011 y Fuller, 2012).

Este mismo comportamiento de varones progenitores controlados y sanos se presentó en los casos de Fernando y Gerardo, quienes a pesar de tener problemas de estrés (colitis, gastritis, síndrome del colon irritable o alguno de ellos) o señalar preocupación por diversos asuntos relacionados con sus hijos, consideraron que su paternidad había sido muy grata. Es importante mencionar que cuando Fernando se enfermó y sintió el compromiso de salir a jugar con su hijo, lo hizo sin comentarle sus molestias, porque no le gusta verle la cara de tristeza de su hijo. En tanto que Gerardo se sostuvo en señalar, que si su hijo se enfermara y él no pudiera hacer nada por encontrarse a varias horas de distancia debido a su trabajo, la esposa tendría que actuar, como si él no tuviera sentimientos de preocupación por la integridad de su hijo. Si fuera el caso, que estos papás no quisieron evidenciar sus vulnerabilidades, quizá sería importante que supieran, que aquellas personas que sufren en silencio, tienen dos veces más probabilidades de padecer hipertensión, enfermedades del corazón o infartos (Laurence, 2009).

Horacio por su parte, recién había cursado porun periodo de desempleo y aunque tenía ahorros para soportarlo por un tiempo y a pesar de que su esposa también aportaba ingresos al hogar, él había experimentado incertidumbre que le produjo complicaciones de salud por un antiguo padecimiento de síndrome del colon irritable y si bien en ese momento podía adjudicarse a la proveeduría económica, este padecimiento tuvo origen diez años antes, en el nacimiento de su primer hijo, cuando tuvo problemas intestinales que condujeron a su hospitalización. Horacio además, verbalizó algunos otros momentos en que se sintió vulnerado con la paternidad, uno de ellos fue cuando por trabajo, tuvo que trasladarse a otra entidad federativa y sólo venir a convivir con su hijo (tenía solo al mayor) los fines de semana. A pesar de tener contacto por videollamada cada noche, ambos vieron disminuido su estado de ánimo, si bien Horacio optó solo por compartir que a su hijo le afectó al disminuir su rendimiento escolar.

Julio fue un hombre al que por poco le impiden ser padre, pero estaba determinado a serlo; lograrlo le ha constado mucho sufrimiento y algunas enfermedades. Se casó a temprana edad, su relación fue complicada y el embarazo, aunque deseado durante diez años, se presentó cuando se estaban divorciando. Desde la óptica de Julio, ella deseaba abortar y él, por "cuidarla", hasta llegó a amarrarla. A los ocho meses de gestación y después de una discusión con Julio, ella intentó suicidarse mediante consumo de pastillas. Por la gravedad, Julio no estuvo en el parto ni lo dejaron interactuar con la niña después, aunque sí fue reprendido por la neonatóloga por la condición en la que llegó su pareja y por ende la bebé.

La esposa de Julio fue dada de alta y la niña permaneció en el hospital para observación. Julio "inhumanamente" la violentaba para extraer leche materna pues ella no quería alimentarla; conducía contrariado por la situación, entregaba la leche en el hospital y regresaba a repetir el proceso para que su hija fuera alimentada a las siguientes tres horas. Este pasaje, Julio lo describe como traumático y en shock, porque no lo había recordado hasta que se lo pregunté. Meses después de haber recibido atención psiquiátrica por el intento de suicidio, la esposa de Julio se fue y se divorciaron.

Julio fue diagnosticado con depresión mayor y medicado por ello, durante los meses siguientes también consumió alcohol, lo suficiente para levantarse diario a trabajar con niños pues era maestro. Su mamá lo apoyaba cuidando a la niña, pero creía que no merecía esa ayuda, lo vivió con mucha culpa. De alguna forma recuperó el ánimo y continuó con su vida a lado de su hija. La llevaba 
al trabajo, tuvo problemas de permisos para atender sus necesidades de salud y hasta le quitaron clases como consecuencia. Julio recibió una educación estricta así que fue duro con su hija, aunque no la agredió físicamente. Se casó por segunda ocasión, pero la hija no se llevaba bien con su pareja y Julio entraba en conflicto, hasta que se divorció por una infidelidad de ella. Julio cree que esa situación le propició el EVC. Permanentemente se sintió culpable por el dolor que le causó a su hija al traerla al mundo y ahora está determinado "a costa de lo que sea" a cuidar de su salud pues tiene bipolaridad, asma e hipotiroidismo; por esta razón, le preocupa su propia salud, pues no quiere morir dejándola sola, pues su red familiar está compuesta por la madre de Julio y él.

\section{Conclusiones}

Una pregunta que tendríamos que intentar contestar a partir de este proyecto es ¿Cómo identificar, nombrar, atender y evitar el deterioro de la salud o la muerte de los varones progenitores? No solamente con el propósito de beneficiarles, sino porque al hacerlo, mejoraríamos también las condiciones de las personas que viven en su entorno inmediato.

Con este proyecto pudimos observar que los papás pudieron ver mermada su salud o puesto en peligro la vida, cuando las funciones como proveedor, protector y educador intervinieron o detonaron una crisis. Pero también, aparecieron nuevos componentes que no habían sido indagados antes, como la posición de la pareja (madre del hijo o la conviviente), mediante la manipulación, el manejo del poder, la infidelidad, por trastornos emocionales o mentales, etc. Es importante resaltar, que no se trata de victimizar o culpar a unos u otras, sino de identificar aquellos elementos que pudieran desgastar la salud física y emocional en este caso de los varones (que también pudieron minar la de las mujeres) y que puedan representar áreas de oportunidad para ser trabajadas desde las políticas públicas.

Otro elemento más fue el enfrentamiento, rechazo o distanciamiento que trazan los hijos hacia el padre, y que en algunos casos impiden el ejercicio y disfrute de la paternidad, además que ubican a los varones en una posición de incomodidad, malestar, inestabilidad emocional y susceptibles al deterioro de la salud o incluso de la muerte en casos extremos.

Un último punto que no podría ignorarse con respecto a este estudio es el ejercicio de violencia, agresión o autoridad excesiva que practican algunos varones sobre uno o todos los miembros de la familia, que pudiera serconfundidos con un comportamiento propio de la construcción de género y no como sugiere Bonino

\footnotetext{
* Se refiere a muertes por ataque al corazón, hemorragia cerebral, infarto o trombosis cerebral, infarto al miocardio o por o por enfermedades al corazón.
}

(2004), con síntomas de malestares prolongados que termina lastimando tanto que pudiera convertirse en enfermedad al no identificarse como un signo de alerta.

Por tal razón se sugiere reforzar la elaboración de conceptos que permitan reconocer los problemas de salud, para poder combatirlos, prevenirlos o atenderlos, y para ello es fundamental la colaboración y voluntad de los profesionales de la salud (Colín, 2020). En Japón, por ejemplo, existen enfermedades como karoshi ${ }^{*}$ y Karojisatsu $^{\dagger}$ que se asocian con la actividad laboral excesiva, por un periodo de tiempo prolongado (Gómez, 2007; Iwasaki, 2006 y Amagasa, et. al., 2005) e incluso el burnout $^{\ddagger}$, ya es reconocido como enfermedad (Tena, 2014) y será integrada formalmente por la OMS, a la Clasificación Internacional de Enfermedades en 2022 (OMC, 2020). Si estos conceptos no se hubieran reconocidos, las causas de muerte por padecimientos del corazón, enfermedades cerebrales y suicidios serían amplias y no se podrían emplear estrategias adecuadas para combatirlas como sí al conocer la relación con la actividad laboral excesiva.

En el caso que aquí presento, propongo una mirada crítica y receptiva en ese mismo sentido, es decir para dialogar y continuar observando comportamientos y prácticas asociadas al género y la paternidad que pudieran ser dañinas y que se puedan nombrar, difundir y tratar, para reducir las muertes evitables y prematura, a la par de descender los índices de violencia dentro del hogar.

También sugiero tratar a la salud con la misma seriedad o interés, que como se trata a la enfermedad. Es decir, el sistema sanitario tiene la responsabilidad de proveer servicios de promoción a la saludy, de prevención y atención de las enfermedades. Pero las intervenciones de promoción tienen basamento en estrategias educativas que son poco funcionales y que promueven conductas individuales, que terminan por sancionar o penalizar socialmente al individuo si no las cumple (Leal y Martínez, 2002). Un ejemplo reciente, es el uso del cubrebocas, la distancia social y el lavado constante de manos, que se estableció en el periodo de pandemia. Aunque las personas lo intentan, muchos no pudieron mantener las recomendaciones por distintas razones y son o fueron cuestionadas socialmente por su falta de cooperación, como si su comportamiento tuviera la intención de dañar y no como sujetos vulnerables.

Me parece que los varones y particularmente los progenitores, cursan por esta misma dificultad. Los percibimos como figuras de referencia, como sujetos privilegiados por su condición de género o incluso como quienes detentan el poder y son violentos, pero son menos las veces que los identificamos como sujetos con

\footnotetext{
$\dagger$ Se refiere a los suicidios vinculados al trabajo.

$\$$ Guarda relación con el creciente esfuerzo de los individuos en el trabajo y que deriva en desinterés laboral y el agotamiento crónico.
} 
necesidades y también vulnerables pues muchas veces no están entrenados para expresar sus emociones y hay que leer las señales que emiten entre líneas al hablar o mediante su comportamiento y actitudes.

La propuesta entonces es apoyar al sector salud a reforzar la valoración del autocuidado y el cuidado del otro, pero no con el propósito responsabilizar al sujeto o cuestionarlo si no lo hace, sino con el fin de ofrecer, herramientas que le sean útiles para tener una mayor oportunidad de conectar con su cuerpo, reconocer las alertas e impedir en la medida de lo posible el deterioro de la salud por situaciones o condiciones evitables, como lo es la construcción del género. Quizá el involucramiento compartido del sector educativo y las instituciones de género pudieran ser una opción para dar origen a nuevas investigaciones que contemplen la salud integral (física y emocional) asociada con lo femenino y masculino en hombres y mujeres potenciales progenitores. Además de estrategias permanentes de difusión de la información, en escuelas, plataformas digitales y otros medios de comunicación y transporte diversos.

A pesar de que la investigación se elaboró con progenitores del género masculino, otra reflexión que logré elaborar, fue que las mujeres pudieran cursar por malestares y tipos de muerte que tampoco se han identificado en el espacio de la maternidad y que superan a lo ya indagado en el certificado de defunción, al incluir igualmente, los asuntos emocionales o mentales que no son atendido de manera prioritaria en los primeros niveles de atención y tampoco por personal médico calificado para identificarlos (Colín, 2021).

\section{Referencias}

Amagasa, T., Nakayama, T., y Takahashi, Y. (2005). Karojisatsu in Japan: characteristics of 22 cases of work-related suicide, Journal of Occupational Health, 47, 157-164.

Bolaños, F. (2014). El grupo de Apoyo Emocional al Desempleo en Hombres: Resultados de Investigación, en Figueroa, Juan Guillermo (Coordinador), Políticas públicas y la experiencia de ser hombre. Paternidad, espacios laborales, salud y educación, El Colegio de México.

Bonino, L. (2004). Masculinidad, salud y sistema sanitario. El caso de la violencia masculina, en Ruíz, Consuelo y Blanco, Pilar (compiladores.), La violencia contra las mujeres: Prevención y detección, Madrid: Díaz Santos.

Cantoral, G. (2014). De las desigualdades de Género a los malestares. La cotidianidad de mujeres y varones de San Cristóbal de las Casas, Chiapas, México [Tesis de doctorado en Ciencias Sociales y Humanísticas, Universidad de Ciencias y Artes de Chiapas].

Caponi, S. (1997). Georges Canguilhem y el estatuto epistemológico del concepto de salud, História, Ciencias, Salud-Manguinbos, IV (2), 287-307.

Colín, Y. (2021). Propuestas metodológicas para robustecer el conocimiento y entendimiento de la salud mental. El caso de algunos hombres en la paternidad, Revista DIVULGARE, Vol. 8, No. $15,24-33$.
Colín, Y. (2020). Salud, enfermedad y muerte de los progenitores desde el lente de algunos profesionales de la salud. En J. Figueroa y A. Salguero (Coord.), Nuevas aristas en el estudio de la paternidad, (pp.511-547), El Colegio de México.

Colín, Y. (2018). Salud, enfermedad y muerte de algunos varones que viven o vivieron la experiencia de la paternidad en la Ciudad de México [Tesis de doctorado en Estudios de Población, El Colegio de México].

De Keijzer, B. (1997). El varón como factor de riesgo: Masculinidad, salud mental y salud reproductiva. En E. Tuñón (coord.), Género y salud en el Sureste de México, ECOSUR Y UJAD, Villahermosa, Veracruz.

Evans, J., Blye, F., Oliffe, J. y Gregory, D. (2011). Health, Illness, Men and Masculinities (HIMM): A theoretical framework for understanding men and their health, JMH review, 8 (1) 7-15.

Figueroa, J. G. (2015). El derecho a la salud en una población que ejerce violencia contra sí misma: el caso de la población masculina, Seminario Los debates sobre la globalización desde una perspectiva de género, Facultad de Filosofía y Letras, UNAM, 24 y 25 de marzo.

Figueroa, J. G. (2014), Entre la paternidad, la salud y la mortalidad: ¿qué nos sugiere una lectura de género? En M. Neves, B. Krimberg y K. C. Kohn (coords.), Caminhos de homens: género e movimentos, Universidad Católica do Rio Grande do Sul, Porto Alegre Brasil, (pp. 297-318).

Figueroa, J. G. (2014a). Algunas propuestas dialógicas para relacionar paternidad, salud y mortalidad, Revista de Ciencias Sociales y Humanidades, Universidad Autónoma Metropolitana Iztapalapa, año 35, (77), 55-75.

Figueroa, J. G. (2011). Paternidad, mortalidad y salud: ¿es posible combinar estos tres términos?, En Estudios sobre Varones y Masculinidades para la generación de políticas públicas y acciones transformadoras, (pp. 71-78), Universidad de la República, Uruguay, Montevideo.

Figueroa, J. G. (2005). Algunos dilemas éticos y políticos al tratar de definir los derechos reproductivos en la experiencia de los varones, Revista Perspectivas Bioéticas, Facultad Latinoamericana de Ciencias Sociales (sede Buenos Aires) y Ediciones SIGNO, vol. 10, (18), 53-75.

Figueroa, J. G. y Nájera, J. (2015). El uso de las autopsias verbales para analizar algunos suicidios de varones progenitores, Acta Universitaria, 25 (3), 19-25. doi: 10.15174/au.2015.848.

Fuller, N. (2012). Repensando el machismo latinoamericano, Masculinities and Social Change, vol. 2, 114-133.

Gómez, M. A. (2007). Masculinidad en la sociedad de riesgo. En L. Jiménez y O. Tena (Coord.), Reflexiones sobre masculinidades y empleo. (pp. 11-20), Universidad Nacional Autónoma de México.

Graña, F. (2011). Raíces de la violencia basada en el género. Los orígenes de la dominación masculina: apuntes para una búsqueda. En Estudios sobre varones y masculinidades para la generación de políticas públicas y acciones transformadoras (pp.103-114), Universidad de la República-Uruguay, Mujer y Salud en Uruguay, Espacio Salud, UNFPA y Naciones Unidas Uruguay.

Iwasaki, K., Takahashi, M. y Nakata, A. (2006). Health Problems due to Long Working Hours in Japan: Working Hours, Workers' Compensation (Karoshi), and Preventive Measures, Industrial Health, 44 (4), 537-540.

Kaufman, M. (1997). Las experiencias contradictorias del poder entre los hombres. En T. Valdés y J. Olavarría (editores) Masculinidad/es, poder y crisis. 63-81, Flacso.

Laurence, J. (2009). Sufrir en silencio puede llevar a un trabajador a la muerte, Periódico La Jornada, miércoles 25 de noviembre de 2009, página 2. 
Le Blanc, G. (2004). Canguilhem y las normas, Buenos Aires, Ediciones Nueva Visión.

Leal, G. y Martínez, C. (2002). Cuatro ensayos sobre calidad clínica de la atención. El caso de la salud reproductiva. En Serie Académicos CBS, Universidad Autónoma Metropolitana, División de ciencias biológicas y de la salud, núm 43, México.

Martínez, C. (2012). El muestreo en investigación cualitativa. Principios básicos y algunas controversias, Universidad Autónoma Metropolitana-Unidad Xochimilco. Ciencias y Salud Colectiva, 17 (3), 613-619.

Martínez, C. (2008). Los múltiples significados de la salud. Un recorrido bajo la guía de Canguilhem. En C. Martínez, (Compiladora), Seis miradas sobre la salud y sus relaciones con el mundo social, UAM-X.

Núñez, G. (2004). Los hombres y el conocimiento. Reflexiones epistemológicas para el estudio de los hombres como sujetos genéricos. En Desacatos Revista de Antropología Social, CIESAS, (15-16), 13-32.

Organización Médica Colegial de España. (2020). La OMS reconoce como enfermedad el burnout o síndrome de estar quemado. Recuperado el 03 de agosto de 2020 de http://www.medicosypacientes.com/articulo/la-omsreconocecomo-enfermedad-elburnout-o-sindrome-de-estarquemado.

Organización Mundial de la Salud. (2021). Definición de salud, https://www.who.int/es/about/who-we-are/frequently-askedquestions\#: :text=\%C2\%BFC\%C3\%B3mo\%20define\%20la\%20 OMS\%20la, ausencia\%20de\%20af ecciones\%200\%20enfermeda des $\%$ C2\%BB. consultado el 03 de abril de 2021

Secretaría de Salud. (2020). Marco normativo y jurídico del Programa de Salud Sexual y Reproductiva de los Adolescentes. https://www.gob.mx/salud/acciones-y-programas/marconormativo-y-juridico-del-programa-de-salud-sexual-yreproductiva-de-los-adolescentes-cnegsr, visitado el 21 de diciembre del 2020.

Tena, O. (2014). Malestares laborales y condición masculina. Reflexiones en torno a la flexibilidad laboral. En J. G. Figueroa, Políticas públicas y la experiencia de ser hombre. Paternidad, espacios laborales, salud y educación, (pp.1-26). El Colegio de México.

Tena, O. (2007). Problemas af ectivos relacionados con la pérdida, disminución y riesgo de pérdida del empleo en varones. En L. Jiménez y O. Tena (coord.), Reflexiones sobre masculinidades y empleo, UNAM.

Torres, L. (2002). Ejercicio de la paternidad en varones de hijos e hijas [Tesis de doctorado en sociología, Universidad Nacional Autónoma de México]. 